\section{DETERGENTS - UNCOMMON HOUSEHOLD POISONS}

Sir,

A 21-year-old man was brought to our Emergency Room in comatose state with labored respiration requiring immediate respiratory and hemodynamic support. His parents disclosed history of ingestion of 150 to $200 \mathrm{~mL}$ of detergent 8 to 10 hours prior to hospitalization. The main chemical content of this detergent was sodium dodecylbenzene, known as iba in local language. Examination revealed tachypnea (Respiratory Rate, 26/min), altered sensorium (E2M4V1) with normal pulse and blood pressure. Oropharyngeal cavity examination showed diffuse ulcerations, but the results of abdominal and cardiovascular examination were within normal limits. There was no focal neurological deficit, and the brainstem reflexes were present. Laboratory investigations revealed normal anion gap (15.7 mEq/L); metabolic acidosis $(\mathrm{pH}-7.29$, $\mathrm{PaO}_{2}-97, \mathrm{PaCO}_{2}-34$, bicarbonates- 16.3 $\mathrm{mEq} / \mathrm{L})$; normal electrolytes; and normal renal and liver functions. Chest $\mathrm{x}$-ray done twice did not show any infiltrates. Initially the patient required controlled-mode ventilation with tidal volume- $10 \mathrm{~mL} / \mathrm{kg} ; \mathrm{FiO}_{2}-100 \%$; PEEP- $5 \mathrm{~cm}$ $\mathrm{H} 2 \mathrm{O}$; and a respiratory rate of $14 / \mathrm{min}$. Upper gastrointestinal endoscopy revealed diffuse hyperemia and multiple ulcers in the esophagus and stomach. A feeding tube was inserted under endoscopic guidance. Supportive care was continued, and the patient improved over the next 5 days; subsequently, he was discharged on proton pump inhibitors. The patient is on regular follow-up, and his feeding tube has been removed.
The American Association of Poison Control Centers reported that $92 \%$ of all toxic exposures occurred at home, and nearly $53 \%$ of these exposures occurred in children less than 6 years of age. ${ }^{[1]}$ Accidental poisoning with detergents can pose a significant risk, especially in children and elderly, but majority of the affected individuals have minimal or no side effects. These detergent products contain anionic surfactants and detergent builders such as sodium phosphate, sodium carbonate and sodium aluminosilicate, which are highly alkaline. Detergents fall into 3 main categories - nonionic, anionic and cationic. Nonionic and anionic detergents are of low toxicity. Cationic detergents, such as benzalkonium chloride and cetrimide, are less frequently encountered in domestic cleaners and produce corrosive effects if a concentrated solution is consumed. The route of exposure is almost always oral ingestion; but inhalational, ocular and dermal exposures and even intraveous route have been reported. [2] Corrosive injury to the gastrointestinal, respiratory and corneal mucosa (depending on route of exposure); cardiac dysfunction; acute renal failure with rhabdomyolysis ${ }^{[3]}$; Acute respiratory distress syndrome (early and late onset); hemolysis; frequent ventricular tachycardia; and coagulation abnormalities have been reported secondary to exposure to household detergents. ${ }^{[2]}$ Our case had evidence of involvement of gastrointestinal and respiratory systems; and altered sensorium, which could have been secondary to hypoxemia. There is no standardized treatment protocol for detergent poisoning. Endotracheal intubation and supportive ventilation are required frequently Syrup of ipecac should not be 
administered, given the risk of aspiration and potential for further caustic injury to the esophagus. This type of household poisoning is common in children due to accidental ingestion because of their curious and exploratory nature. Our case is unique as it is the first reported case of adult exposure to detergent poisoning from India.

\section{SURI, S. MAHI, A. BHALLA, N. SHARMA, S. VARMA \\ Department of Internal Medicine, Post Graduate Institute of Medical Education and Research, Chandigarh, India \\ Correspondence: Dr. Vikas Suri Department of Internal Medicine, PGIMER, Chandigarh - 160 012, India E-mail: surivikas9479@gmail.com}

\section{REFERENCES}

1. Litovitz TL, Klein-Schwartz W, White S, Cobaugh DJ, Youniss J, Omslaer JC, et al. 2000 Annual report of the American Association of Poison Control Centers Toxic Exposure Surveillance System. Am J Emerg Med 2001;19:337-95.

2. Okumura T, Suzuki K, Yamane K, Kumada K, Kobayashi R, Fukuda A, et al. Intravenous detergent poisoning. J Toxicol Clin Toxicol 2000;38:347-50.

3. Prabhakar KS, Pall AA, Woo KT. Rhabdomyolysis and acute renal failure complicating detergent ingestion. Singapore Med J 2000;41:182-3.

DOI: 10.4103/0019-5359.55115 